\title{
Nephrology and intervention in the community - On the need for improving the interplay between nephrology centers and primary care in Portugal
}

\author{
Idalina Beirão ${ }^{1,2}$, Edgar Almeida ${ }^{3,4}$ \\ ${ }^{1}$ Institute of Biomedical Sciences Abel Salazar, Porto University, Portugal \\ ${ }^{2}$ Nephrology Department, University Hospital Center of Porto, Portugal \\ ${ }^{3}$ Faculty of Medicine, University of Lisbon; \\ ${ }^{4}$ Nephrology Department, Beatriz Ângelo Hospital, Loures, Portugal
}

\section{ABSTRACT}

Chronic kidney disease is increasing in prevalence, especially among older people. The development of integrated strategies between primary health care and hospital care is crucial to curb the growth of this pathology, already considered a public health problem. Telemedicine approaches the different levels of care and creates a great confidence in managing CKD in the community, with a beneficial and real patient-centered care. Other strategies that lead to empowerment of the primary care medical doctors should also be considered. This manuscript summarizes some of these aspects and lists the benefits of its implementation in Portugal.

Key-Words: telemedicine, nephrology, chronic kidney disease. Primary Care, renal disease management

\section{TELEMEDICINE IN NEPHROLOGY, by Idalina Beirão}

Chronic kidney disease (CKD) is the cause of noncommunicable disease mortality that has increased most since 1990 (data from 188 countries), with great impact on patients' morbidity and mortality, as well as having an enormous economic impact. The incidence of CKD increases with age, but the recognition of the disease by the population is still very low and, consequently, its detection is late ${ }^{1}$. In United States, the costs related to CKD continue to increase especially for pre-dialysis patients ${ }^{2}$, and a similar picture occurs in our country.

Telemedicine is part of the telehealth universe directed to clinical services that have been suggested as an improvement in the care of patients with chronic kidney disease. Electronic consultations referred by primary care doctors, with sharing of patients' electronic health records with nephrologists, allow timely access to helpful advice, avoid unnecessary referrals to nephrologists and create a great confidence in managing CKD in the community, with a beneficial and real patient-centered care $^{3}$. As expected, telemedicine provided time and cost savings when compared to usual care, with positive results in participants' satisfaction and only a little increase in staff hours expended, related to telemedicine ${ }^{4}$. It also proved to improve blood glucose control in diabetic patients and to a lesser degree, blood pressure control in hypertension patients, two of the most frequent chronic conditions associated to $\mathrm{CKD}^{5}$.
Portugal has the highest incidence of CKD in Europe and the incidence and prevalence of advanced CKD requiring renal replacement therapy are higher than other occidental European countries. In a study of patients referred to the nephrology outpatient clinic at Centro Hospitalar Universitário do Porto during 2012, 49\% of the new referred patients were older than 65 years of age, with many comorbidities, namely hypertension (96\%), diabetes mellitus (50\%), coronary artery disease $(25 \%)$, cerebrovascular disease $(24 \%)$, and $38 \%$ of them had functional dependence ${ }^{6}$. The high prevalence of CKD in an aging population, with multiple comorbidities and mobility difficulties implies changes in policy healthcare, with strategies to prevent and control the disease development and adjustment of human resources. Thus, a closer articulation between primary prevention (primary care) and secondary prevention (hospital care) will optimize strategies in patient management and improve the prevention and treatment of CKD.

The use of telemedicine allows a faster and early assessment by nephrology and, in some cases may prevent patient displacement to the hospital and all related costs, namely personal, family and/or caregivers and healthcare transports. Additionally, it allows the reduction of the hospital visits with reduction of the waiting list.

Under Portuguese law, teleconsultation is considered defined as a medical consultation, as part of of telemedicine, carried out at a distance using the use of interactive, audiovisual and data 
communications with mandatory registration in the clinical process of the patient. Different modalities, namely teleconsulting in deferred time, teleconsulting in real time and urgent teleconsultation are planned. These different modalities aim at increasing disease prevention and the articulation with Primary Care, increasing the adequate and timely approach and optimization of resources management.

\section{WHY WE NEED A BETTER INTERPLAY BETWEEN NEPHROLOGY CENTERS AND PRIMARY CARE, by Edgar Almeida}

The international comparisons provided by the United States Renal Data System ${ }^{7}$ underline that Portugal has higher incidence and prevalence of end-stage renal disease compared to Spain, a country which shares a common genetic and demographic origin, a similar gross domestic product, a similar diet, and a similar organization of and access to a health care system. The reasons for this panorama are not fully understood although poor control of risks factors is one of the most frequently proposed ${ }^{8}$, which means that the role of primary care is fundamental to reduce the risks and for early detection of renal disease.

Over the last 25 years, several achievements have changed dramatically how nephrologists deal with CKD, such the publication of the Modification of Diet in Renal Disease (MDRD) study in 1994 and the proposition of the MDRD equation for creatinine based estimation of glomerular filtration rate (1999). Later, a stratification of severity of CKD into five stages was proposed (2002) and the KDIGO initiative launched (2005). More recently, albuminuria was included in the stratification of CKD. A substantial number of current primary care doctors graduated more than 25 years ago and are unaware of these improvements. A quiz given to primary care doctors attending a seminar on CKD revealed that only $42 \%$ responded correctly to a very simple question about the stages of CKD. In a sample of 108 requesting files for outpatient consultation of Nephrology at the Hospital Beatriz Ângelo (Loures, Portugal), $27.7 \%$ omitted to say if the patient was hypertensive (or under anti-hypertensive medication); $50.9 \%$ said nothing about diabetic condition (yes or no, well controlled or no); $25 \%$ omitted serum creatinine value and $62.6 \%$ omitted to say if the patient had proteinuria in the dipstick. These data reinforce the need to empower primary care doctors in this area, with new guidelines to diagnose early detection in high-risk patients, stratification and prognostication of CKD. The new instruments for estimating GFR equations generate information on CKD stages that should be carefully interpreted. Age is the most important determinant of eGFR, which means that people over 85 years will all have a reduced renal function.
However, this does not mean that CKD will progress to ESRD as death is a powerful competitive risk. Recently, in a longitudinal study with a mean follow-up of 3.7 years, we demonstrated that patients with macroalbuminuria and patients with congestive heart failure are at increased risk for progression toward ESRD. In this study, a subset of patients aged 75 years or more progressed toward ESRD if they were diabetics and had proteinuria 9 .

In conclusion, empowering primary care doctors means that they are able to make an adequate selection which will improve the referral pattern to nephrology centers. In this scenario, nephrology centers can focus their attention on patients with high rate of disease progression, with the confidence that other patients are adequately supervised by primary care doctors. A better interplay with nephrology centers through communication channels such as telephone, e-mail or telemedicine will empower primary care doctors to provide adequate nephroprotection to our shared patients.

Disclosure of potential conflicts of interest: none declared.

\section{References}

1. Luyckx VA, Tonelli M, Stanifer JW. The global burden of kidney disease and the sustainable development goals. Bull World Health Organ. 2018;96(6):414-422D.

2. Saran R, Li Y, Robinson B, Agodoa LY, Ayanian J, Bragg-Gresham J, et al. US Renal Data System 2015 Annual Data Report: epidemiology of kidney disease in the United States. Am J Kidney Dis. 2016; 67: Svii.

3. Stoves J, Connolly J, Cheung CK, Grange A, Rhodes P, O'Donoghue D, et al. Electronic consultation as an alternative to hospital referral for patients with chronic kidney disease: a novel application for networked electronic health records to improve the accessibility and efficiency of healthcare. Qual Saf Health Care. 2010;19(5): e54.

4. Campbell M, Akbari A, Amos S, Keyes C. Feasibility of providing nephrology services to remote communities with videoconferencing. J Telemed Telecare. 2012; 18(1): 13-16.

5. Hailey D. Telehealth in Nephrology Care - Promises and Challenges. Am J Kidney Dis. 2016; 68(1): 5-7.

6. Lascasas JS, Fonseca I, Malheiro J, Santos S, Campos A, Castro A, et al. Demographic, clinical characteristics and cardiovascular disease burden in a Portuguese cohort of older chronic kidney disease patients. J Bras Nefrologia. 2019; 41(1): 29-37.

7. United State Renal Data System. www.usrds.org (accessed August 21th, 2018).

8. Stel VS, Brück K, Fraser S, Zoccali C, Massy ZA and Jager KJ. International differences in chronic kidney disease prevalence: a key public health and epidemiological research issue. Nephrol Dial Transpl. 2017; 32: ii129-ii135.

9. Falcão L, Fernandes S, Raimundo M, Costa A, Teixeira C, Silva S, et al. MP 384 Which CKD stage 3 patients should be referred to a Nephrologist? Results of a retrospective, patient level, cohort analysis. Nephrol Dial Transpl. 2017; 32, (suppl_3), iii568-iii569.

\section{Correspondence to:}

Idalina Beirão MD, PhD.

Nephrology Department, Centro Hospitalar Universitário do Porto, Porto. Portugal

E-mail: idalina.m.b@gmail.com 